\title{
P-0855: Relationship of adiposity indices with cardio-metabolic and inflammatory markers among urban and rural adolescents
}

\author{
M. Chopra ${ }^{1}$, A. Siddhu', D. Bharadwaj2, N. Tandon ${ }^{3}$ \\ ${ }^{1}$ Lady Irwin College, Food and Nutrition, New Delhi, India
}

2Jawaharlal Nehru University, Systems Genomics Laboratory- School of Biotechnology, New Delhi, India ${ }^{3}$ All India Institute of Medical Sciences, Endocrinology and Metabolism, New Delhi, India

\section{INTRODUCTION}

- Rates of childhood and adolescent obesity have increased worldwide and obesity is associated with metabolic derangements and subclinical inflammation.

- In the Indian context, with ongoing socio-demographic and nutritional transition, prevalence of obesity and associated chronic diseases are on the rise, with higher body fat content reported at lower BMI levels, as compared to non-Hispanic whites and Caucasians.

- Changes in hormones, body composition and adverse lifestyle among adolescents makes them extremely vulnerable to develop obesity and associated metabolic diseases.

\section{OBJECTIVES}

1. To assess the levels of adiposity indices, cardiometabolic risk factors and markers of inflammation among apparently healthy urban and rural Indian adolescents.

2. To determine relationship of adiposity indices with elevated levels of cardio-metabolic and inflammatory markers.

\section{METHODS}

Study design and sample size: Cross-sectional study on 2484 school-going adolescents (11-17 years).

Locale: Urban area $(n=1790 ; 9$ schools; Delhi) and rural areas ( $n=694 ; 3$ schools; Harsaru village, District Gurgaon, Haryana and Duhai village, District Ghaziabad, Uttar Pradesh) of north India.

\section{Data Collection}

- Anthropometric measurements: Height (anthropometric rod), weight (digital balance) and classification of adolescents based on weight status (overweight/obese [OW/OB] and normal [NW]) using BMI for age and gender IOTF standards. Waist (WC) and hip circumference (inelastic tape) and computation of waist to hip ratio (WHR) and waist to height ratio (WHtR).

- Body composition measurements: Fat $\%$ and fat mass (TANITA Body Composition Analyser, BC-420 MA).

- Blood Pressure (BP): Systolic and diastolic BP (OMRONSEM-1 automated machine, using appropriate cuff size). - Cardio-metabolic variables: Fasting blood samples of glucose, total cholesterol (TC), LDL-C, HDL-C, triglyceride (TG) and markers of inflammation including CRP, TNFalpha $(n=231)$, IL-6 $(n=377)$, leptin $(n=383)$, resistin $(n=381)$ and adiponectin $(n=378)$.

\section{Statistical Analysis}

- Median (IQR) and percentages were computed for continuous and categorical variables.

- To test for difference among categories, Mann-Whitney $U$ test and chi-square test for continuous and categorical variables were used.

- Adiposity indices: BMI, WC, WHR, WHtR, fat\% and fat mass were divided into tertiles (tertile 3-high risk; tertile 1-reference group).

- Logistic regression to estimate odds ratio (OR) of individual elevated cardio-metabolic risk factors with adiposity indices (tertiles), adjusting for age, gender and location, using SPSS software version 20.

Systolic BP/diastolic BP $\geq 95$ th percentile for height, age, gender, $\mathrm{TC} \geq 200 \mathrm{mg} / \mathrm{dL}$, LDL-c $\geq 130 \mathrm{mg} / \mathrm{dL}$, HDL-c $<40 \mathrm{mg} / \mathrm{dL}, \mathrm{TG} \geq 130 \mathrm{mg} / \mathrm{dL}$, fasting glucose $\geq 126 \mathrm{mg} / \mathrm{dL}$.

Markers of inflammation (higher than median value; CRP $>0.4 \mathrm{mg} / \mathrm{L}$; TNFalpha> $31.2 \mathrm{pg} / \mathrm{mL} ; \quad$ IL- $6>3.5 \mathrm{pg} / \mathrm{mL}$; leptin $>2.6 \mathrm{ng} / \mathrm{mL} ;$ resistin $>7.4 \mathrm{ng} / \mathrm{mL}$; lower than median value, adiponectin $<2625.5 \mathrm{pg} / \mathrm{mL}$ ) derived from the study sample. Inflammatory markers data were on a sample subset (except CRP), hence missing values were replaced with median values for regression analysis.

\section{RESULTS}

- Significantly higher median values of adiposity indices, systolic and diastolic BP TC, LDL-C, CRP, TNF-alpha, leptin and lower levels of fasting glucose and adiponectin were observed among urban as compared to rural adolescents ( $\mathbf{p}$ values $<0.001-0.019)$.

- Prevalence (\%) of adverse cardio-metabolic risk factors among urban and rural adolescents is given in Figure 1.

\section{Figure 1: Prevalence (\%) of adverse cardio-metabolic risk factors among adolescents}

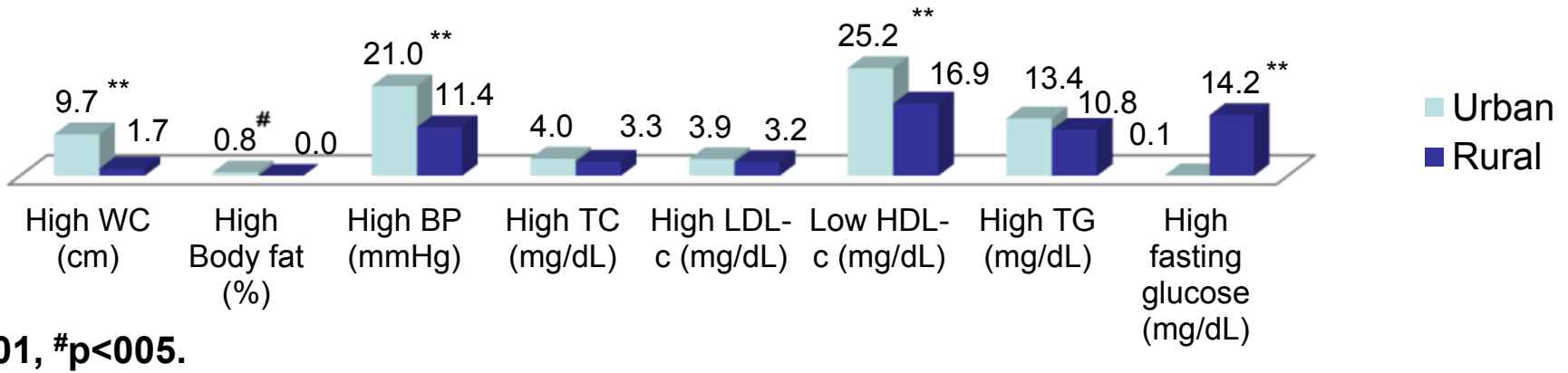

p<0.001, ${ }_{0}<005$

Table 1 as given below shows adjusted OR for elevated cardio-metabolic variables by adiposity indices (tertiles).

\begin{tabular}{|c|c|c|c|c|c|c|}
\hline & $\begin{array}{l}\text { High BP } \\
(\mathrm{mmHg})\end{array}$ & $\begin{array}{l}\text { High TC } \\
\text { (mg/dL) }\end{array}$ & $\begin{array}{l}\text { High LDL-c } \\
\text { (mg/dL) }\end{array}$ & $\begin{array}{c}\text { Low HDL-c } \\
(\mathrm{mg} / \mathrm{dL})\end{array}$ & $\begin{array}{l}\text { High TG } \\
(\mathrm{mg} / \mathrm{dL})\end{array}$ & $\begin{array}{l}\text { High fasting } \\
\text { glucose } \\
\text { (mg/dL) }\end{array}$ \\
\hline $\begin{array}{l}\text { BMI } \\
\left(\mathrm{Kg} / \mathrm{m}^{2}\right)\end{array}$ & $\begin{array}{c}0.95 \\
(0.52,1.74)\end{array}$ & $\begin{array}{c}0.70 \\
(0.17,2.81)\end{array}$ & $\begin{array}{c}1.49 \\
(0.35,6.38)\end{array}$ & $\begin{array}{c}1.22 \\
(0.70,2.12)\end{array}$ & $\begin{array}{c}2.24^{*} \\
(1.08,4.62)\end{array}$ & $\begin{array}{c}0.56 \\
(0.16,1.98)\end{array}$ \\
\hline $\begin{array}{l}\text { WC } \\
(\mathrm{cm})\end{array}$ & $\begin{array}{c}0.92 \\
(0.58,1.45)\end{array}$ & $\begin{array}{c}2.53^{*} \\
(1.01,7.06)\end{array}$ & $\begin{array}{c}1.72 \\
(0.60,4.95)\end{array}$ & $\begin{array}{c}1.42^{*} \\
(1.02,2.14)\end{array}$ & $\begin{array}{c}1.49^{*} \\
(1.01,2.57)\end{array}$ & $\begin{array}{c}3.35^{*} \\
(1.24,9.03)\end{array}$ \\
\hline Fat\% & $\begin{array}{c}1.05 \\
(0.63,1.73)\end{array}$ & $\begin{array}{c}3.03 \\
(0.93,9.84)\end{array}$ & $\begin{array}{c}5.24^{\star *} \\
(1.54,17.8)\end{array}$ & $\begin{array}{c}1.01 \\
(0.63,1.61)\end{array}$ & $\begin{array}{c}1.43 \\
(0.78,2.64)\end{array}$ & $\begin{array}{c}1.11 \\
(0.30,4.17)\end{array}$ \\
\hline $\begin{array}{l}\text { Fat mass } \\
(\mathrm{Kg})\end{array}$ & $\begin{array}{c}1.40 \\
(0.79,2.47)\end{array}$ & $\begin{array}{c}2.01 \\
(0.52,7.75)\end{array}$ & $\begin{array}{c}1.40 \\
(0.36,5.40)\end{array}$ & $\begin{array}{c}0.98 \\
(0.571 .66)\end{array}$ & $\begin{array}{c}1.40 \\
(0.69,2.84)\end{array}$ & $\begin{array}{c}1.17 \\
(0.30,4.5)\end{array}$ \\
\hline WHR & $\begin{array}{c}1.59^{*} \\
(1.03,2.71)\end{array}$ & $\begin{array}{c}1.80 \\
(0.60,5.46)\end{array}$ & $\begin{array}{c}1.41 \\
(0.44,4.53)\end{array}$ & $\begin{array}{c}1.49^{*} \\
(1.04,2.36)\end{array}$ & $\begin{array}{c}1.62 \\
(0.89,2.93)\end{array}$ & $\begin{array}{c}2.69^{*} \\
(1.11,7.56)\end{array}$ \\
\hline WHtR & $\begin{array}{c}2.63^{*} \\
(1.24,5.57)\end{array}$ & $\begin{array}{c}0.37 \\
(0.07,1.86)\end{array}$ & $\begin{array}{c}0.30 \\
(0.06,1.62)\end{array}$ & $\begin{array}{c}1.21 \\
(0.62,2.35)\end{array}$ & $\begin{array}{c}0.46^{*} \\
(0.19,0.98)\end{array}$ & $\begin{array}{c}0.85 \\
(0.20,3.61)\end{array}$ \\
\hline \multicolumn{7}{|c|}{ 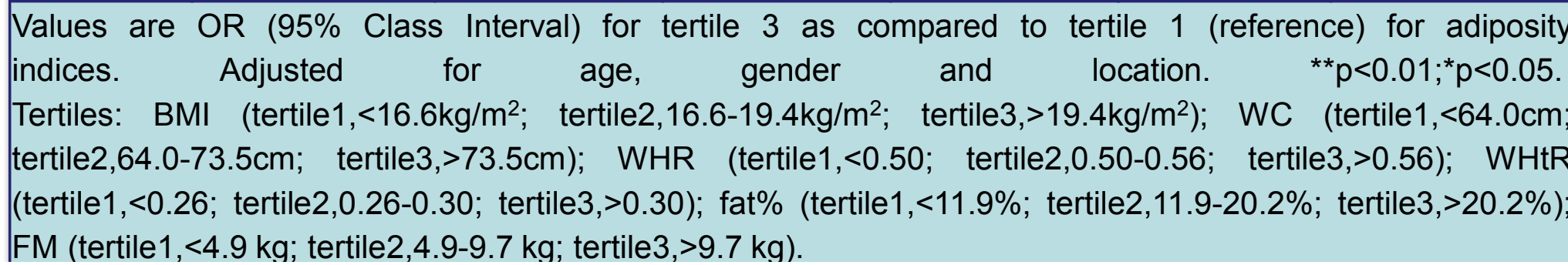 } \\
\hline
\end{tabular}

Figure 2 as given below shows adjusted OR for elevated inflammatory markers by adiposity indices (tertiles).

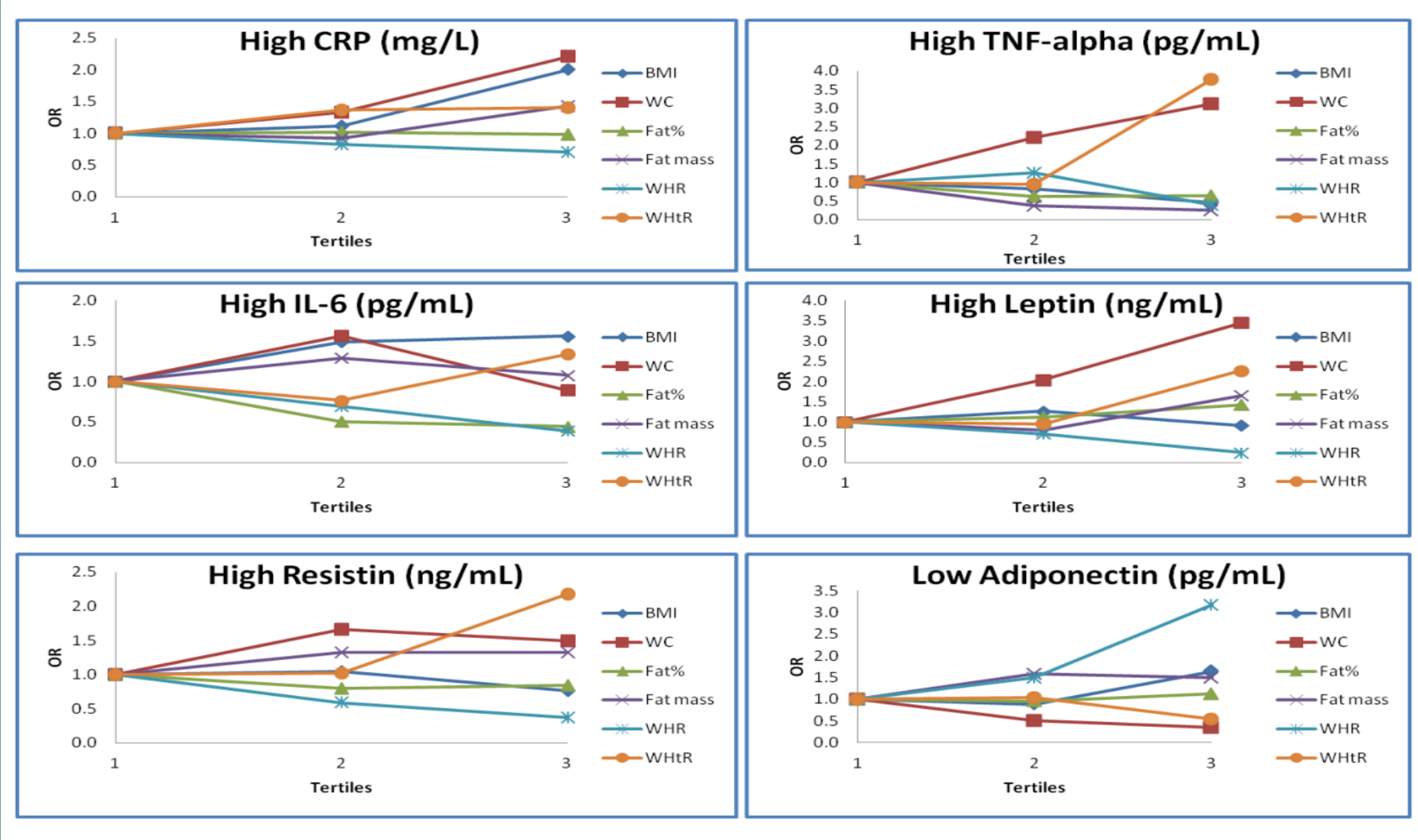

\section{DISCUSSION}

Significantly higher levels of adiposity indices, body composition, cardio-metabolic and inflammatory markers were obtained among urban as compared to rural adolescents. For most of the adiposity indices, an increase in odds at tertile 3 was observed as compared to tertile 1 with cardio-metabolic and inflammatory markers, highlighting the relationship between adiposity and cardio-metabolic risk, even among adolescents. 